\title{
Crocetin protects against fulminant hepatic failure induced by lipopolysaccharide/D-galactosamine by decreasing apoptosis, inflammation and oxidative stress in a rat model
}

\author{
KE GAO ${ }^{1}$, FAQUAN LIU ${ }^{2}$, XI CHEN ${ }^{3}$, MENGXUE CHEN $^{2}$, QINGWEN DENG ${ }^{2}$, \\ XINGJIAN ZOU ${ }^{2}$ and HONGXING GUO ${ }^{2}$ \\ Departments of ${ }^{1}$ Pathology and ${ }^{2}$ Gastroenterology, The Fifth Affiliated Hospital, Southern Medical University, \\ Guangzhou, Guangdong 510900; ${ }^{3}$ Department of Ears, Nose and Throat, The Third \\ People's Hospital of Shenzhen, Shenzhen, Guangdong 518115, P.R. China
}

Received October 9, 2018; Accepted July 5, 2019

DOI: $10.3892 /$ etm.2019.8030

\begin{abstract}
Fulminant hepatic failure (FHF) is a clinical syndrome characterized by sudden and severe liver dysfunction. Apoptosis and inflammation are essential for the pathogenesis of FHF. Crocetin, the major component present in saffron, has been reported to possess anti-inflammatory and antioxidant functions; however, its role in FHF is poorly understood. The aim of this study was to explore the protective effects of crocetin against lipopolysac $\$$ charide (LPS)/D-galactosamine (D-GalN)-induced FHF and the underlying mechanisms in a rat model. For the in vivo study, rats were assigned to the LPS/D-GalN group or to the crocetin pre-treatment+LPS/DGalN group. Each group was then further divided according to the different LPS/D-GalN treatment times of $0,6,12$ or $48 \mathrm{~h}$. The results demonstrated that crocetin pre-treatment efficiently protected against LPS/D-GalN-induced FHF by improving liver tissue morphology, reducing total bilirubin generation and decreasing the activities of alanine transaminase and aspartate aminotransferase. Moreover, crocetin pre-treatment significantly decreased hepatocyte apoptosis, p53 mRNA expression and the expression of proteins in the caspase family and the Bcl-2 pro-apoptotic family following LPS/D-GalN treatment. Furthermore, crocetin also decreased the secretion of pro-inflammatory cytokines in the serum and in the liver via suppression of $\mathrm{NF}-\kappa \mathrm{B}$ activation, and also suppressed hepatic oxidative stress. In conclusion, crocetin protected against LPS/D-GalN-induced FHF and inhibited apoptosis, inflammation and oxidative stress. The underlying
\end{abstract}

Correspondence to: Dr Hongxing Guo or Dr Faquan Liu, Department of Gastroenterology, The Fifth Affiliated Hospital, Southern Medical University, 566 Congcheng Road, Guangzhou, Guangdong 510900, P.R. China

E-mail: hongxg82@sina.com

E-mail: liufq1015@163.com

Key words: lipopolysaccharide/D-galactosamine, fulminant hepatic failure, crocetin, apoptosis, inflammation mechanisms may be related to the regulation of apoptotic proteins in the caspase family and the Bcl-2 family, as well as the modulation of NF- $\kappa \mathrm{B}$ expression. Therefore, crocetin may be used as a novel therapy for preventing FHF.

\section{Introduction}

The liver is vulnerable to various factors, such as bacteria, hepatitis viruses, alcohol, hepatotoxic drugs and oxidative products leading to hepatic failure $(1,2)$. Fulminant hepatic failure (FHF) is a severe clinical syndrome characterized by massive hepatocyte apoptosis with a high mortality rate (60-80\%) (3). The high mortality rate of acute liver failure often requires liver transplantation (4). Although liver transplantation is a highly successful treatment, it is severely limited by the shortage in donor organs (5). Therefore, identifying an effective medical therapy is important. D-Galactosamine (D-GalN) and lipopolysaccharide (LPS)-induced liver injury is a well-established experimental model that closely resembles human fulminant hepatitis in both morphological and functional features (6). Increasing evidence demonstrates that inflammatory responses are important pathogenic factors that contribute to LPS/D-GalN-induced FHF (7). D-GalN is a specific hepatotoxic agent that increases the lethal effects of LPS (8). Stimulation by LPS can trigger the Toll-like receptor 4 signalling pathway and can activate $\mathrm{NF}-\kappa \mathrm{B}$ to release pro-inflammatory cytokines such as tumour necrosis factor- $\alpha$ (TNF- $\alpha$ ), interleukin (IL)- $1 \beta$ and IL-6, which have pivotal roles in the pathogenesis of LPS/D-GalN-induced acute hepatitis (9). Therefore, inhibiting inflammation may be a potential preventive measure for the development of FHF.

Crocetin, the major component present in saffron, is a 20 -carbon chain molecule containing six double bonds with a carboxylic acid group at each end $(10,11)$. In the past few decades, an increasing amount of evidence has identified that crocetin has promising anti-inflammatory, antioxidant and neuroprotective properties (12). Investigation into the hepatoprotective effects of crocetin determined that crocetin administration increased the survival of rats during resuscitation post-haemorrhage (13) and protected against $\mathrm{CCl}_{4}$-induced 
liver damage (14). However, its role in FHF remains poorly elucidated.

Therefore, to better understand the effects of crocetin on FHF, the present study established an FHF model using LPS/D-GalN and evaluated hepatic apoptosis and inflammation following crocetin pre-treatment.

\section{Materials and methods}

Animals and their diets. A total of 60 male Wistar rats (63-70 days old; 180-200 g) and their food were obtained from Guangdong Medical Laboratory Animal Centre. All rats were maintained in a 12-h light/dark cycle at a constant temperature and humidity $\left(22 \pm 3^{\circ} \mathrm{C}\right.$ and $50 \pm 20 \%$, respectively) with ad libitum access to food and water.

Experimental protocol. Animals were randomly assigned to one of the three following groups with 20 rats per group: i) Control; ii) LPS/D-GalN; or iii) crocetin+LPS/D-GalN. Each group was then further divided into four subgroups according to the time of assessment: 0, 6, 12 or $48 \mathrm{~h}$. Animals in the LPS/D-GalN group received an intraperitoneal injection of $300 \mathrm{mg}$ of D-GalN per $\mathrm{kg}$ of body weight (Sigma-Aldrich; Merck $\mathrm{KGaA}$ ) and then were injected intradermally with $50 \mathrm{mg}$ of LPS per $\mathrm{kg}$ of body weight (Sigma-Aldrich; Merck KGaA) (15). Rats were sacrificed after 0, 6, 12 and $48 \mathrm{~h}$ after LPS/D-GalN injection. Animals in the crocetin+LPS/D-GalN group were pre-treated with $200 \mu \mathrm{l}$ of $5 \mu \mathrm{mol} / 1$ crocetin once, (Sigma-Aldrich; Merck KGaA) $(16,17)$ 1 day before the injection of LPS/D-GalN. Furthermore, no treatment was performed on the rats in the Control group. All the rats were anaesthetized with avertin $(250 \mathrm{mg} / \mathrm{kg})$ and sacrificed by exsanguination from the femoral artery. Liver and $100 \mu \mathrm{l}$ blood samples were collected for subsequent analysis. All rats received humane care according to the Guidelines for the Care and Use of Research Animals established by Southern Medical University and the experimental protocol was approved by the Ethics Committee of the Fifth Affiliated Hospital of Southern Medical University.

Haematoxylin and eosin $(H \& E)$ staining. Livers were fixed in 4\% paraformaldehyde solution for 24-36 $\mathrm{h}$ at room temperature. The fixed samples were embedded in paraffin. Microtome sections were prepared (5 $\mu \mathrm{m}$ thickness) and stained with H\&E according to Xin et al (18). The histology images (magnification, x100 and x400) were captured using a light microscope (Shanghai Optical Instrument Factory No. 1).

Biochemical evaluation of serum. After centrifugation for $10 \mathrm{~min}$ at $4^{\circ} \mathrm{C}(300 \mathrm{x} \mathrm{g}$; Centrifuge 5804R), the activities of alanine transaminase (ALT), aspartate aminotransferase (AST) and total bilirubin (TBIL) in the rat serum were determined using an automatic biochemical blood analyser (cat. no. 7600-210; Hitachi High-Technologies Corporation). IL-6 (cat. no. ml002828), IL-1 $\beta$ (cat. no. ml003549) and TNF- $\alpha$ (cat. no. ml002859) concentrations were detected using commercial ELISA kits (Shanghai Enzyme-linked Biotechnology Co., Ltd.) according to the manufacturer's instructions. The activities of malondialdehyde (MDA; cat. no. A003-1-2) and superoxide dismutase (SOD; cat. no. A001-3-2) were detected using commercial kits from
Table I. Primers used for reverse transcription-quantitative PCR.

\begin{tabular}{ll} 
Gene & \multicolumn{2}{c}{ Primer sequence } \\
\hline IL-1 $\beta$ & F: 5'-AGCATCCAGCTTCAAATCTC-3' \\
& R: 5'-AGCTCATGGAGAATACCACT-3' \\
TNF- $\alpha$ & F: 5'-TGAAGTAGTGGCCTGGATTGC-3' \\
& R: 5'-GACATTCCGGGATCCAGTGA-3' \\
iNOS & F: 5'-GAGCAAAAAAGGGCAACAC-3' \\
& R: 5'-CGCACTTCTGTCTCTCCAAA-3' \\
p53 & F: 5'-GAGCTGACAAGACAATGCTAG-3' \\
& R: 5'-TCATACGATCTGTATCCTCCAG-3' \\
GAPDH & F: 5'-CCCATTCTTCCACCTTTGAT-3' \\
& R: 5'-CAACTGAGGGCCTCTCTCTT-3'
\end{tabular}

IL-1 $\beta$, interleukin-1 $\beta$; TNF- $\alpha$, tumour necrosis factor- $\alpha$; iNOS, inducible nitric oxide synthase; $F$, forward; $R$, reverse.

Nanjing Jiancheng Bioengineering) according to the instructions of the manufacturer.

Reverse transcription-quantitative PCR (RT-qPCR). Total RNA was extracted using TRIzol ${ }^{\circledR}$ (Invitrogen; Thermo Fisher Scientific, Inc.) according to the manufacturer's instructions. Quantitative and qualitative analyses of isolated RNA were assessed from the ratio of absorbance at 260 and $280 \mathrm{~nm}$ by a Biophotometer Plus (Eppendorf) and 1\% agarose gel electrophoresis. Complementary DNA was synthesized from $1 \mu \mathrm{g}$ of total RNA using the commercial Moloney Murine Leukaemia Virus reverse transcriptase kit (Promega Corporation) according to the manufacturer's instruction. Primer sequences for the rat genes were designed and selected using Primer 5.0 (Premier Biosoft International) and Oligo 7.0 software (Molecular Biology Insights, Inc.), as presented in Table I. GAPDH was used as a housekeeping gene to normalize target gene transcript levels. qPCR was performed using SYBR-Green qPCR Super Mix (Invitrogen; Thermo Fisher Scientific, Inc.) and the ABI StepOne Real-Time PCR System or the 7500 Fast Real-Time PCR System (Applied Biosystems; Thermo Fisher Scientific, Inc.). The thermocycling conditions were as follows: $2 \mathrm{~min}$ at $50^{\circ} \mathrm{C}, 2 \mathrm{~min}$ at $95^{\circ} \mathrm{C}$, followed by 40 cycles of $15 \mathrm{sec}$ denaturation at $95^{\circ} \mathrm{C}, 32 \mathrm{sec}$ annealing/extension at $60^{\circ} \mathrm{C}$ then a final melting curve analysis to monitor the purity of the PCR product. The $2^{-\Delta \Delta C q}$ method was used to quantify mRNA abundance (19). Relative gene expression levels were normalized to GAPDH.

Western blot analysis. Liver tissue lysates were prepared by adding $10 \mu \mathrm{l}$ phenylmethylsulfonyl fluoride and $10 \mu \mathrm{l}$ protease inhibitor cocktail (Sigma-Aldrich; Merck KGaA) into $1 \mathrm{ml}$ radioimmunoprecipitation assay lysis buffer (Beyotime Institute of Biotechnology). Liver tissues (100 mg) were resuspended in $500 \mu \mathrm{l}$ cell lysis buffer and were homogenized and rocked for $30 \mathrm{~min}$ on ice. Crude lysates were then centrifuged at $12,000 \mathrm{x} \mathrm{g}$ for $10 \mathrm{~min}$ at $4^{\circ} \mathrm{C}$. Equal amounts $(20 \mu \mathrm{g})$ of protein from each sample were subjected to $10 \%$ SDS-PAGE and then proteins on the gel were transferred to polyvinylidene difluoride membranes 
A
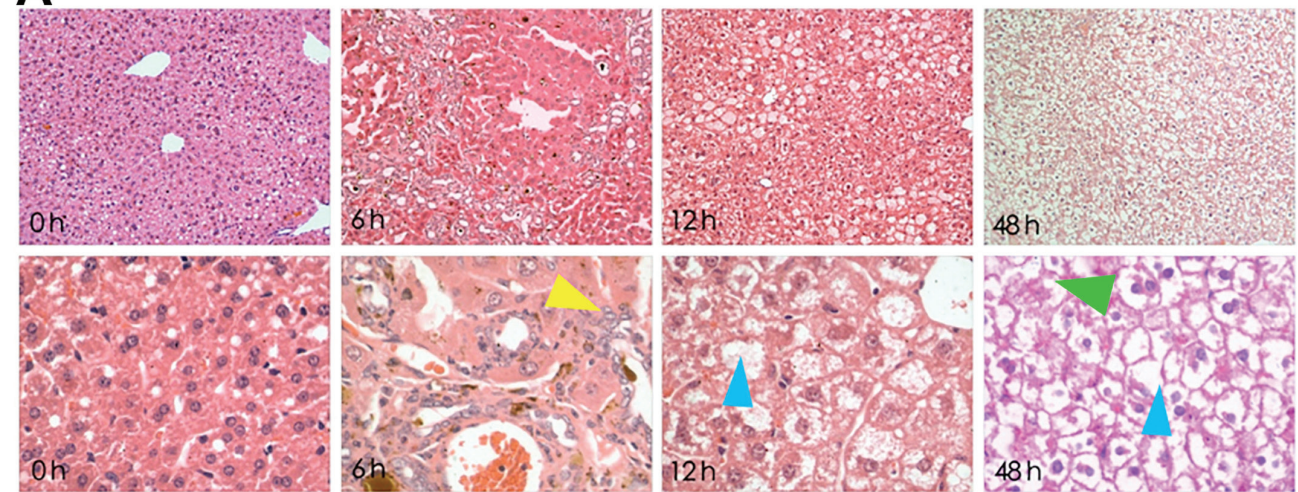

B
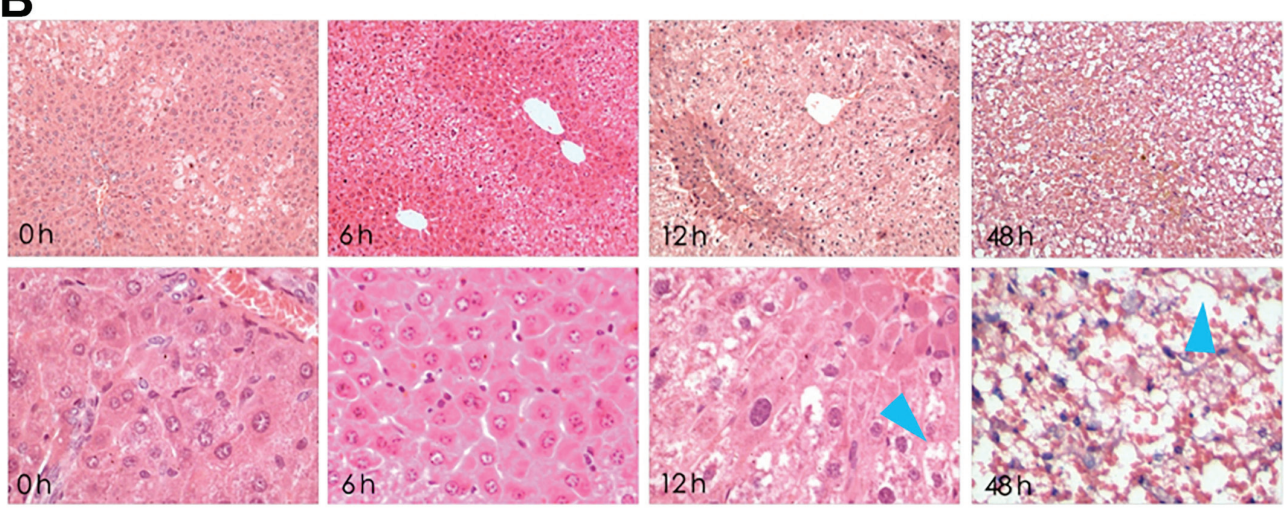

Figure 1. Crocetin pre-treatment attenuates the effect of LPS/D-GalN on the liver. (A) Microscopic alterations in rat livers after receiving LPS/D-GalN for 0,6, 12 and $48 \mathrm{~h}$ (magnification, x100 upper panels and x400 lower panels). (B) Microscopic alterations in the livers of rats pre-treated with crocetin before LPS/D-GalN induction for 0, 6, 12 and $48 \mathrm{~h}$ (magnification, x100 upper panels and x400 lower panels). Yellow arrows highlight inflammatory cell infiltration, blue arrows highlight necrotic area and green arrows highlight haemorrhage. LPS, lipopolysaccharide; D-GalN, D-galactosamine.

(EMD Millipore). Membranes were blocked with 5\% skim milk at room temperature for $1 \mathrm{~h}$ and were then incubated with the primary antibodies anti-GAPDH $(1: 1,000$; cat. no. EPR16891; Abcam), anti-caspase-8 (1:3,000; cat. no. ab32125; Abcam), anti-truncated BH3 interacting domain death agonist (tBid) (1:1,000; cat. no. sc-56025; Santa Cruz Biotechnology, Inc.), anti-caspase-12 (1:2,000; cat. no. ab62484; Abcam), anti-BCL2 like 11 (Bim) (1:500; cat. no. ab32158; Abcam), anti-caspase-9 (1:1,000; cat. no. ab32539; Abcam), anti-caspase-3 (1:500; cat. no. ab13847; Abcam), anti-Bax (1:1,000; cat. no. ab32503; Abcam), anti-NF-кB (1:500; cat. no. ab194729; Abcam) overnight at $4^{\circ} \mathrm{C}$. After washing with Tris-buffered saline and polysorbate 20 , membranes were incubated with the secondary antibodies conjugated to horseradish peroxidase (SouthernBiotech), including Goat Anti-Rabbit IgG (H+L) (1:20,000; cat. no. 4050-05) and Rabbit Anti-Mouse IgG (H+L)-HRP (1:10,000; cat. no. 6170-05). The blots were then developed with an enhanced chemiluminescence detection system (ChemiDoc MP; Bio-Rad Laboratories, Inc.) according to the manufacturer's instructions. Densitometric quantification of band intensities was determined using Image J software (National Institutes of Health).

Flow cytometry. An Annexin V-fluorescein isothiocyanate (FITC) Apoptosis Detection kit was utilized to detect early apoptosis [Annexin V-FITC ${ }^{+} /$propidium iodide (PI)-; Q4], late apoptosis (Annexin $\mathrm{V}$-FITC ${ }^{+} / \mathrm{PI}^{+}$; Q2), and necrosis (Annexin V-FITC-/PI'; Q1) according to the manufacturer's instructions (Nanjing KeyGen Biotech. Co. Ltd.). Briefly, fresh liver was removed and the liver mononuclear was immediately isolated. Isolation of liver mononuclear cells was achieved by cutting the organ into small pieces and then grinding samples with glass rod on a 200 mesh (200 holes/cm) stainless steel cell strainer. Following sufficient grinding, the cells were collected by centrifugation at $300 \mathrm{x} \mathrm{g}$ for $5 \mathrm{~min}$ at $4^{\circ} \mathrm{C}$. Cells were washed twice with PBS to obtain the purified hepatocytes. Subsequently, cells were digested with $0.25 \%$ trypsin and collected by centrifugation at $300 \mathrm{xg}$ for $5 \mathrm{~min}$ at $4^{\circ} \mathrm{C}$. After being washed twice with PBS, the cells were stained with Annexin V-FITC for $15 \mathrm{~min}$ and PI for $5 \mathrm{~min}$ at room temperature. The apoptotic cells were identified by flow cytometry (LSRFortessa; BD Biosciences).

Statistical analysis. Statistical analysis was performed using the SPSS 18.0 software (SPSS, Inc.) and the figures were produced with GraphPad Prism 5.0 software (GraphPad Software, Inc.). All data are presented as the mean \pm standard deviation. Differences between groups were examined using one-way analysis of variance with Tukey's post-hoc test. $\mathrm{P}<0.05$ was considered to indicate a statistically significant difference.

\section{Results}

Crocetin attenuates LPS/D-GalN-induced FHF in the liver. Histological slides were stained with $\mathrm{H} \& \mathrm{E}$ and were analysed at x100 and x400 magnification (Fig. 1). In the LPS/D-GalN 

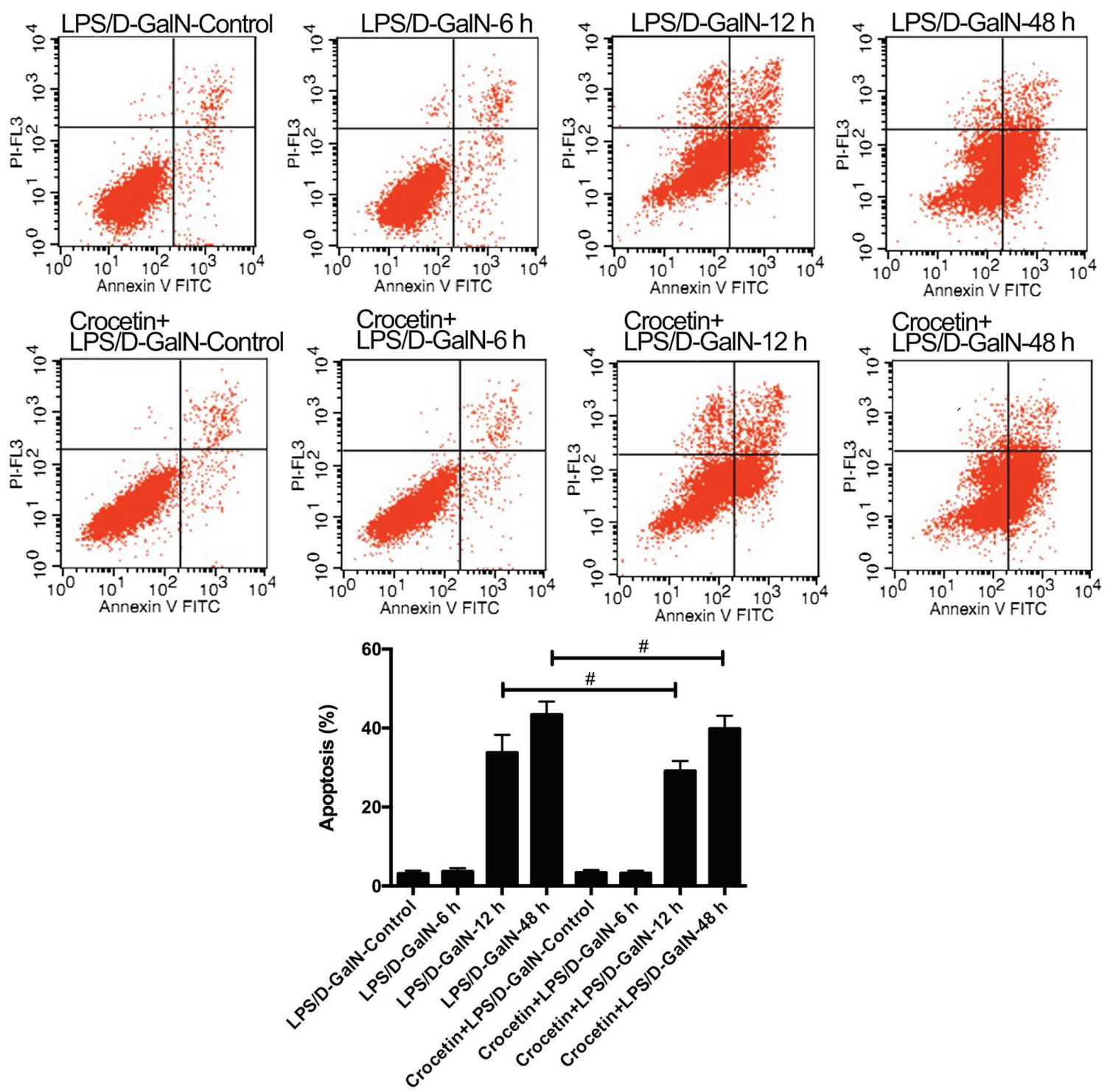

Figure 2. Crocetin pretreatment reduces apoptosis in LPS/D-GalN-induced fulminant hepatic failure. Representative flow cytometry plots and quantification of apoptosis. ${ }^{\text {P }}$ <0.05 LPS, lipopolysaccharide; D-GalN, D-galactosamine; PI, propidium iodide; FITC, fluorescein isothiocyanate.

groups, severe liver pathological changes were characterized by deformation and irregular arrangement, but these alterations were attenuated with crocetin pre-treatment. In detail, 6-h LPS/D-GalN treatment induced acidophilic changes, inflammatory infiltration and few apoptotic bodies. The 12-h LPS/D-GalN treatment induced nuclear dissolution, nuclear fragmentation, massive haemorrhagic necrosis and massive apoptotic bodies. Finally, 48-h LPS/D-GalN treatment induced a fibre mesh stent collapse in addition to massive haemorrhagic necrosis and apoptotic bodies (Fig. 1A). However, preservation of liver tissue following 6 and 12-h LPS/D-GalN induction was observed in crocetin-pre-treated rats. However, 48-h LPS/D-GalN stimulation following crocetin pretreatment still led to eosinophilic changes and inflammatory infiltration with nuclear pyknosis and apoptotic features (Fig. 1B).

Crocetin decreases the activities of ALT and AST and reduced the level of TBIL in serum. Exposure to LPS/D-GalN was associated with significant increases in the liver damage markers ALT, AST and TBIL at all assessment times ( $\mathrm{P}<0.01$; Table II).
The highest values of these parameters were detected following LPS/D-GalN treatment for $48 \mathrm{~h}$. Pre-treatment with crocetin significantly decreased the activities of ALT, AST and TBIL at all the assessment times compared to their counterpart LPS/D-GalN groups $(\mathrm{P}<0.01$; Table II).

Crocetin regulates LPS/D-GalN-induced hepatocyte apoptosis. The percentage of apoptotic cells was augmented in rats receiving LPS/D-GalN in a time-dependent manner (Fig. 2). Crocetin pre-treatment significantly decreased cell apoptosis following LPS/D-GalN treatment for 12 and $48 \mathrm{~h}(\mathrm{P}<0.01$; Fig. 2). To further detect the anti-apoptotic ability of crocetin, the expression levels of p53 and apoptosis-related proteins were measured in the LPS/D-GalN and the crocetin+LPS/D-GalN groups following $48 \mathrm{~h}$ of stimulation (Fig. 3). The results demonstrated that p53 mRNA levels and the expression levels of apoptosis-related proteins, including caspase-3, -8, -9 and -12 , tBID, Bim, and BAX, were significantly upregulated in the livers of rats following LPS/D-GalN treatment $(\mathrm{P}<0.01)$. With crocetin treatment, both the p53 level $(\mathrm{P}<0.01$; Fig. $3 \mathrm{~A})$ and the 
Table II. Crocetin decreases the LPS/D-GalN-induced activities of ALT and AST and the level of TBIL.

\begin{tabular}{|c|c|c|c|c|c|c|c|}
\hline \multirow[b]{2}{*}{ Parameters } & \multirow[b]{2}{*}{ Control } & \multicolumn{3}{|c|}{ LPS/D-GalN } & \multicolumn{3}{|c|}{ Crocetin+LPS/D-GalN } \\
\hline & & $12-\mathrm{h}$ & 24-h & 48-h & $12-\mathrm{h}$ & 24-h & $48-\mathrm{h}$ \\
\hline ALT, IU/l & $43.38 \pm 7.23$ & $526.34 \pm 96.54^{\mathrm{a}}$ & $1131.23 \pm 198.76^{a}$ & $1973.24 \pm 206.21^{\mathrm{a}}$ & $165.34 \pm 52.08^{b}$ & $511.47 \pm 62.79^{b}$ & $1001.27 \pm 137.55^{b}$ \\
\hline AST, IU/1 & $59.01 \pm 8.17$ & $467.89 \pm 93.91^{\mathrm{a}}$ & $927.89 \pm 79.58^{\mathrm{a}}$ & $1047.54 \pm 185.74^{\mathrm{a}}$ & $136.67 \pm 20.33^{b}$ & $324.22 \pm 48.07^{\mathrm{b}}$ & $847.05 \pm 68.51^{\mathrm{b}}$ \\
\hline TBIL,$\mu \mathrm{mol} / 1$ & $0.68 \pm 0.25$ & $2.65 \pm 0.53^{\mathrm{a}}$ & $36.46 \pm 6.63^{\mathrm{a}}$ & $61.73 \pm 9.71^{\mathrm{a}}$ & $1.55 \pm 1.04^{\mathrm{b}}$ & $12.35 \pm 5.02^{\mathrm{b}}$ & $51.78 \pm 8.61^{\mathrm{b}}$ \\
\hline
\end{tabular}

Data are expressed as the mean $\pm \mathrm{SD}(\mathrm{n}=8)$. ${ }^{\mathrm{a}} \mathrm{P}<0.01$ vs. control group; ${ }^{\mathrm{b}} \mathrm{P}<0.01$ vs. LPS/D-GalN group. LPS, lipopolysaccharide; D-GalN, D-galactosamine; ALT, alanine transaminase; AST, aspartate aminotransferase; TBIL, total bilirubin.
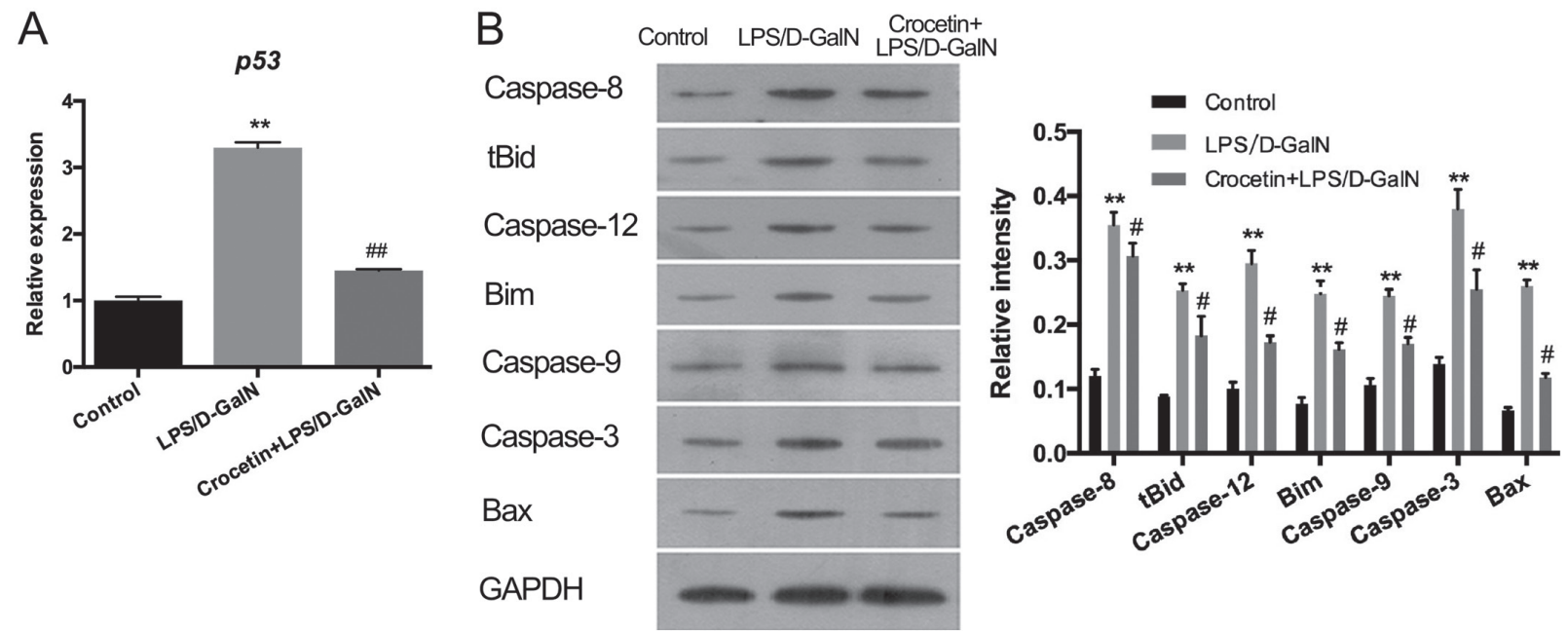

Figure 3. Crocetin pre-treatment decreases pro-apoptotic protein expression in LPS/D-GalN-induced fulminant hepatic failure. (A) mRNA expression of p53 and (B) the expression of apoptosis-related proteins in LPS/D-GalN-treated rats. Data are expressed as the mean $\pm \mathrm{SD}(\mathrm{n}=3)$. ${ }^{* *} \mathrm{P}<0.01 \mathrm{vs}$. respective control group; ${ }^{\#} \mathrm{P}<0.05$ and ${ }^{\# \#} \mathrm{P}<0.01$ vs. respective LPS/D-GalN group. LPS, lipopolysaccharide; D-GalN, D-galactosamine; tBid, truncated BH3 interacting domain death agonist; Bim, BCL2 like 11.

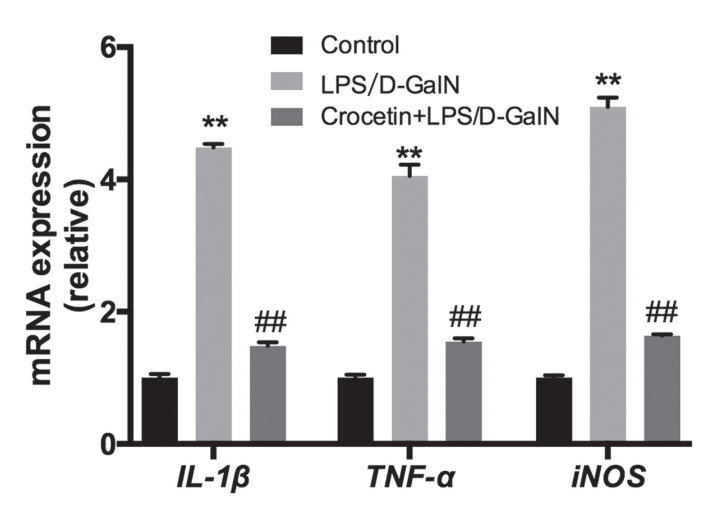

Figure 4. Crocetin pre-treatment decreases the expression of genes related to inflammation in LPS/D-GalN-induced fulminant hepatic failure. Data are expressed as the mean $\pm \mathrm{SD}(\mathrm{n}=3){ }^{* * *} \mathrm{P}<0.01$ vs. respective control group; ${ }^{\# \#} \mathrm{P}<0.01$ vs. respective LPS/D-GalN group. LPS, lipopolysaccharide; D-GalN, D-galactosamine; IL-1 $\beta$, interleukin-1 $\beta$; TNF- $\alpha$, tumour necrosis factor- $\alpha$; iNOS, inducible nitric oxide synthase.

apoptotic protein levels $(\mathrm{P}<0.05$; Fig. 3B) were significantly decreased.

Crocetin decreases LPS/D-GalN-induced inflammation. Following 48-h LPS/D-GalN treatment, the liver inflammation level was investigated. mRNA expression levels of IL-1 $\beta$, TNF- $\alpha$ and inducible nitric oxide synthase (iNOS) were significantly increased in the LPS/D-GalN treatment group ( $\mathrm{P}<0.01$; Fig. 4). Conversely, IL-1 $\beta$, TNF- $\alpha$ and iNOS mRNA levels were significantly reduced $(\mathrm{P}<0.01)$ in the crocetin+LPS/D-GalN treatment group (Fig. 4). ELISA data demonstrated that serum and hepatic IL-1 $\beta$, TNF- $\alpha$ and IL-6 concentrations were significantly elevated in rats receiving LPS/D-GalN $(\mathrm{P}<0.05)$ but significantly decreased by crocetin treatment $(\mathrm{P}<0.05$; Fig. 5). Moreover, hepatic NF- $\kappa \mathrm{B}$ expression was significantly increased with LPS/D-GalN treatment $(\mathrm{P}<0.05)$ but was significantly decreased following crocetin administration $(\mathrm{P}<0.05 ;$ Fig. 6). The results of the current study indicated that crocetin could decrease LPS/D-GalN-induced hepatic inflammation.

Crocetin decreases LPS/D-GalN-induced oxidative stress. As demonstrated in Table III, LPS/D-GalN treatment resulted in higher malondialdehyde $(\mathrm{MDA})$ levels $(\mathrm{P}<0.01)$ and lower superoxide dismutase (SOD) activities in the liver $(\mathrm{P}<0.01)$ compared with the control group. By contrast, compared to the LPS/D-GalN group, crocetin significantly decreased the MDA concentration $(\mathrm{P}<0.01)$ and increased the SOD activity $(\mathrm{P}<0.05)$. 

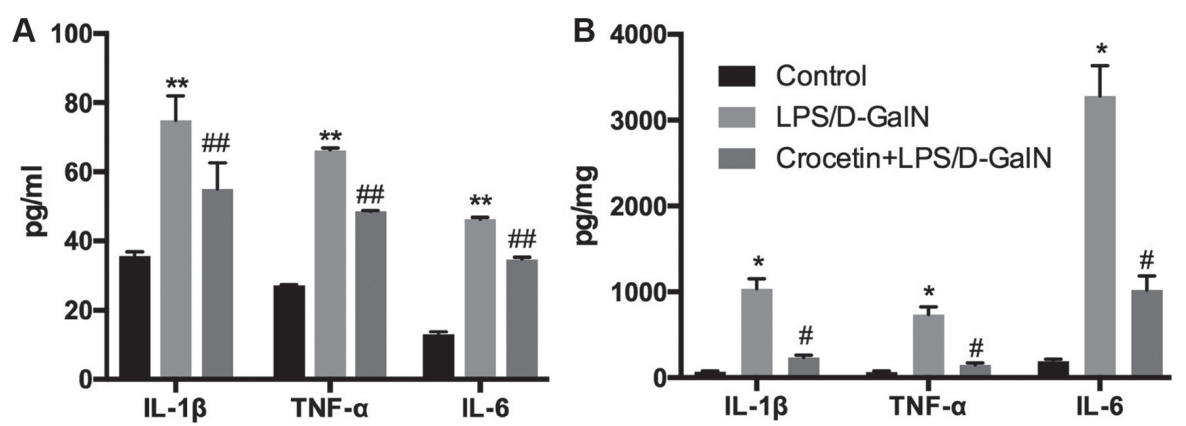

Figure 5. Crocetin pre-treatment decreases the levels of inflammatory factors in LPS/D-GalN-induced fulminant hepatic failure. (A) IL-1 13 , TNF- $\alpha$ and IL-6 in the serum and (B) livers of LPS/D-GalN-treated rats at $48 \mathrm{~h}$. Data are expressed as the mean $\pm \mathrm{SD}(\mathrm{n}=3)$. ${ }^{*} \mathrm{P}<0.05$ and ${ }^{* * *} \mathrm{P}<0.01$ vs. respective control group; ${ }^{\#} \mathrm{P}<0.05$ and ${ }^{\# \#} \mathrm{P}<0.01$ vs. respective LPS/D-GalN group. LPS, lipopolysaccharide; D-GalN, D-galactosamine; IL-1 $\beta$, interleukin-1 $\beta$; TNF- $\alpha$, tumour necrosis factor- $\alpha$; IL-6, interleukin- 6 ; NF- $\kappa B$, nuclear factor- $\kappa$ B.
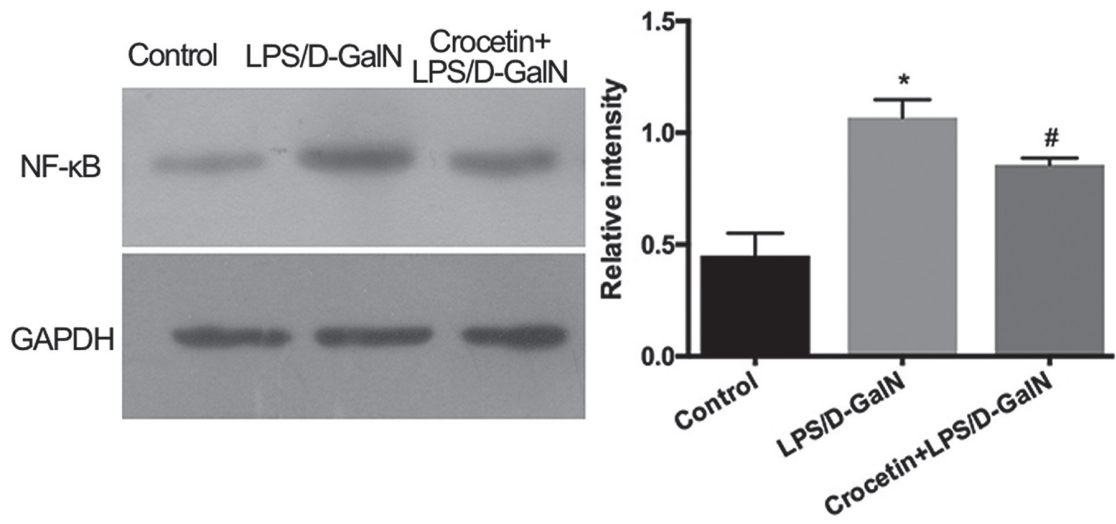

Figure 6. Effects of crocetin pre-treatment on the hepatic expression of NF-kB in LPS/D-GalN-treated rats at $48 \mathrm{~h}$. Data are expressed as the mean $\pm \mathrm{SD}(\mathrm{n}=3$ ). ${ }^{*} \mathrm{P}<0.05$ vs. control group; ${ }^{*} \mathrm{P}<0.05$ vs. LPS/D-GalN group. LPS, lipopolysaccharide; D-GalN, D-galactosamine.

Table III. Crocetin decreases LPS/D-GalN-induced oxidative stress.

\begin{tabular}{lcr}
\hline Group & $\begin{array}{c}\text { MDA, } \\
\mathrm{nmol} / \mathrm{mg}\end{array}$ & $\begin{array}{c}\text { SOD, } \\
\mathrm{U} / \mathrm{mg}\end{array}$ \\
\hline Control (n=6) & $15.03 \pm 1.09$ & $127.32 \pm 15.71$ \\
LPS/D-GalN (n=6) & $126.39 \pm 15.26^{\mathrm{a}}$ & $63.26 \pm 6.58^{\mathrm{a}}$ \\
Crocetin+LPS/D-GalN (n=6) & $58.37 \pm 5.18^{\mathrm{c}}$ & $82.07 \pm 8.33^{\mathrm{b}}$ \\
\hline
\end{tabular}

Data are expressed as the mean $\pm S D(n=6)$. ${ }^{a} P<0.01$ vs. control group; ${ }^{\mathrm{P}} \mathrm{P}<0.05$ and ${ }^{\mathrm{c}} \mathrm{P}<0.01$ vs. LPS/D-GalN group. LPS, lipopolysaccharide; D-GalN, D-galactosamine; MDA, malondialdehyde; SOD, superoxidase dismutase.

\section{Discussion}

The LPS/D-GalN-induced animal model of FHF is strongly relevant to human liver failure and has been widely used to investigate the mechanisms of and potential therapeutic drugs for clinical FHF (20). As anticipated, the present results confirmed that LPS/D-GalN stimulation for 6,12 and $48 \mathrm{~h}$ induced severe liver damage, which manifested as altered liver morphology, increased TBIL levels and enhanced ALT and AST activities. The pathological condition of severe liver injury is characterised by significant elevations of ALT, AST and TBIL in the serum (21-24). However, crocetin pre-treatment effectively improved the structural integrity of parenchymal hepatocytes and decreased these biochemical indicators for liver injury, evidencing the protective effect of crocetin against FHF.

Apoptosis is a complicated biological process that regulates fulminant hepatic cell division and death $(14,25)$. In the present study, apoptotic hepatocytes were detected using flow cytometry. The results demonstrated that the number of apoptotic cells was increased by LPS/D-GalN treatment in a time-dependent manner, whereas crocetin significantly decreased the number of apoptotic cells following LPS/D-GalN treatment for 12 and $48 \mathrm{~h}$. Furthermore, the tumour suppressor p53 is a key protein in preventing cell transformation and tumour progression. Activated by a variety of stimuli, $\mathrm{p} 53$ regulates cell cycle arrest and apoptosis (26). In the present study, the elevated hepatic p53 expression in rats that had received LPS/D-GalN for $48 \mathrm{~h}$ was decreased following crocetin pre-treatment. In addition, massive activation of caspases is a crucial process during the induction of apoptosis and is associated with the pathogenesis of acute hepatic failure (27). Caspase- 8 triggers the extrinsic apoptotic pathway, whilst caspase- 9 triggers the intrinsic pathway (28). Caspase-12 is an endoplasmic reticulum (ER)-specific caspase that is specifically activated by disturbances to ER homeostasis (29). Caspase-3, the predominant downstream effector, can be activated by caspase- $8,-9$ and -12 (30). In addition, the Bcl-2 family proteins BAX, Bim and 
tBID (the activated form of BID) translocate to the mitochondria and mediate the permeabilization of the outer membrane, thereby facilitating apoptosis $(31,32)$. In the present study, western blot analysis demonstrated that the expression of caspase-3, $-8,-9$ and -12 , BAX, Bim and tBID was upregulated following LPS/D-GalN treatment, but crocetin effectively downregulated the expression of all the pro-apoptotic proteins. Taken together, the aforementioned findings suggested that crocetin can attenuate apoptosis by decreasing the expression of p53 and pro-apoptotic proteins.

Inflammation is considered to be a strongly interrelated biological event involved in the pathogenesis of FHF (33). Previous studies have demonstrated that LPS/D-GalN-induced FHF was accompanied by the release of multiple pro-inflammatory cytokines, including TNF- $\alpha$, IL-1 $\beta$ and IL-6, from liver cells $(1,33)$. The present study determined that crocetin pre-treatment effectively decreased the expression of hepatic TNF- $\alpha$ and IL-1 $\beta$ as well as reducing the secretion of serum TNF- $\alpha$, IL-1 $\beta$ and IL-6 in an FHF rat model. In addition, NO is a highly reactive oxidant produced by parenchymal and nonparenchymal liver cells from L-arginine via the action of iNOS (34). In animal models, NO synthases are involved in the pathogenesis of hyperdynamic circulation (35-38), and the role of iNOS in causing liver damage in FHF has been demonstrated in LPS/D-GalN-treated animals $(38,39)$. In the present study, increased iNOS mRNA expression was observed in the LPS/D-GalN group, whilst crocetin treatment dramatically downregulated the hepatic iNOS level. The expression of iNOS is tightly regulated by the transcriptional factor $\mathrm{NF}-\kappa \mathrm{B}$, which promotes the secretion of cytokines including TNF- $\alpha$, IL- $1 \beta$ and IL- 6 and has a pivotal role in animals models of FHF $(40,41)$. Therefore, the present study analysed $\mathrm{NF}-\kappa \mathrm{B}$ expression in the liver. In line with the cytokine level results, crocetin pre-treatment also significantly inhibited the NF- $\kappa$ B level in rats following LPS/D-GalN stimulation. Taken together, the present findings indicated that crocetin attenuated inflammation in an FHF rat model by decreasing the generation of cytokines by inhibiting NF- $\kappa \mathrm{B}$.

FHF can induce oxidative stress (42). Therefore, the oxidative stress level in the liver was analysed. MDA is a lipid peroxidation product and SOD is a well-studied antioxidant enzyme (43). In the present study, LPS/D-GalN treatment increased the MDA levels and decreased the SOD activity. By contrast, crocetin pre-treatment reduced MDA generation and elevated SOD activity. These results indicated that crocetin may ameliorate oxidative stress caused by LPS/D-GalN.

In conclusion, the present study demonstrated the beneficial effect of crocetin against LPS/D-GalN-induced FHF where it decreased the secretion of pro-inflammatory cytokines via inhibition of $\mathrm{NF}-\kappa \mathrm{B}$, whilst also attenuating hepatocellular apoptosis and oxidative stress. Thus, crocetin may be a potential and promising agent for preventing FHF.

\section{Acknowledgements}

Not applicable.

\section{Funding}

The present study was supported by the Traditional Chinese Medicine Bureau Research Project of Guangdong Province (grant no. 20171175) and Natural Scientific Fund of Guangdong Province (grant no. 2018A030310479).

\section{Availability of data and materials}

All data generated or analyzed during this study are included in this published article.

\section{Authors' contributions}

$\mathrm{KG}, \mathrm{XC}$ and $\mathrm{MC}$ were responsible for performing the experiments. QD and XZ were responsible for data analysis and manuscript preparation. FL and $\mathrm{HG}$ were responsible for manuscript writing and revision and experimental design. All authors read and approved the final manuscript.

\section{Ethics approval and consent to participate}

The experimental protocols were approved by the Ethics Committee of The Fifth Affiliated Hospital of Southern Medical University.

\section{Patient consent for publication}

Not applicable.

\section{Competing interests}

The authors declare that they have no competing interests.

\section{References}

1. El-Agamy DS, Makled MN and Gamil NM: Protective effects of agmatine against D-galactosamine and lipopolysaccharide-induced fulminant hepatic failure in mice. Inflammopharmacology 22: 187-194, 2014.

2. Zhang X, Ding J, Gou C, Wen T, Li L, Wang X, Yang H, Liu D, Lou J, Cehn D, et al: Qingchangligan formula attenuates the in inflammatory response to protect the liver from acute failure induced by d-galactosamine/lipopolysaccharide in mice. J Ethnopharmacol 201: 108-116, 2017.

3. Lee SB, Kang JW, Kim SJ, Ahn J, Kim J and Lee SM: Afzelin ameliorates Dgalactosamine and lipopolysaccharide-induced fulminant hepatic failure by modulating mitochondrial quality control and dynamics (150/150). Brit J Pharmacol 174: 195-209, 2017.

4. Marzio HD and Sass DA: Fulminant hepatic failure: Diagnosis and management. Springer International Publishing, Switzerland, pp229-245, 2014.

5. Nasralla D, Coussios CC, Mergental H, Akhtar MZ, Butler AJ, Ceresa CD, Chiocchia V, Dutton SJ, Garcia-Valdecasas JC, Heaton $\mathrm{N}$, et al: A randomized trial of normothermic preservation in liver transplantation. Nature 557: 50-56, 2018.

6. Duan GJ, Zhu J, Xu CY, Wan JY, Zhang L, Ge XD, Liu LM and Liu YS: Protective effect of Go6976, a PKD inhibitor, on LPS/d-GalN-induced acute liver injury in mice. Inflamm Res 6: 357-366, 2011.

7. Jaeschke H: Reactive oxygen and mechanisms of inammatory liver injury. J Gastroenterol Hepatol 7: 718-724, 2000.

8. Ben Ari Z, Avlas O, Pappo O, Veacheslav Z, Cheporko Y, Bachmetov L, Zemel R, Asher S, Sharon E, Grief F and Hochhauser E: Reduced hepatic injury in toll-like receptor 4-deficient mice following D-galactosamine/lipopolysaccharide induced fulminant hepatic failure. Cell Physiol Biochem 29: 41-50, 2012.

9. Kim SJ, Kim JK, Lee DU, Kwak JH and Lee SM: Genipin protects lipopolysaccharide-induced apoptotic liver damage in D-galactosamine-sensitized mice. Eur J Pharmacol 635: 188-193, 2010. 
10. Bolhassani A, Khavari A and Bathaie SZ: Saffron and natural carotenoids: Biochemical activities and anti-tumor effects. Biochem Biophys Acta 1845: 20-30, 2014.

11. Cao W, Cui J, Li S, Zhang D, Guo Y, Li Q, Luan Y and Liu X: Crocetin restores diabetic endothelial progenitor cell dysfunction by enhancing NO bioavailability via regulation of PI3K/AKT-eNOS and ROS pathways. Life Sci 181: 9-16, 2017.

12. Zhuang X, Dong A, Wang R and Shi A: Crocetin treatment inhibits proliferation of colon cancer cells through down-regulation of genes involved in the inflammation. Saudi J Biol Sci 25: 1767-1771, 2018

13. Yang R, Vernon K, Thomas A, Morrison D, Qureshi N and Van Way CW III: Crocetin reduces activation of hepatic apoptotic pathways and improves survival in experimental hemorrhagic shock. JPEN J Parenter Enteral Nutr 35: 107-113, 2011.

14. Chen P, Chen Y, Wang Y, Cai S, Deng L, Liu J and Zhang H: Comparative evaluation of hepatoprotective activities of geniposide, crocins and crocetin by CCl4-induced liver injury in mice. Biomol Ther (Seoul) 24: 156-162, 2016.

15. Liu LM, Zhang JX, Luo J, Guo HX, Deng H, Chen JY and Sun SL: A role of cell apoptosis in lipopolysaccharide (LPS)-induced nonlethal liver injury in D-galactosamine (D-GalN)-sensitized Rats. Digest Dis Sci 53: 1316-1324, 2008.

16. Gao K, Guo H, Liu L, Ding Y, Kuang M and Li J: Liver protection of crocetin against paraquat poisoning in rats. Chin Crit Care Med 28: 876-880, 2016 (In Chinese).

17. Guo H, Gao K, Zou X, Deng Q, Chen M and Liu F: Crocetin promotes autophagy in injured rat hepatocytes induced by lipopolysaccharide and D-galactosamine in vitro. Nan Fang Yi Ke Da Xue Xue Bao 38: 1121-1125, 2018 (In Chinese).

18. Xin J, Zeng D, Wang H, Ni X, Yi D, Pan K and Jing B: Preventing non-alcoholic fatty liver disease through Lactobacillus johnsonil BS15 by attenuating inflammation and mitochondrial injury and improving gut environment in obese mice. Appl Microbiol Biotechnol 98: 6817-6829, 2014

19. Livak KJ and Schmittgen TD: Analysis of relative gene expression data using real-time quantitative PCR and the 2(-Delta Delta C(T)) method. Methods 25: 402-408, 2001

20. Lin X, Zhang S, Huang R, Tan S, Liang S, Wu X, Zhuo L and Huang Q: Protective effect of tormentic acid from Potentilla chinensis against lipopolysaccharide/D-galactosamine induced fulminant hepatic failure in mice. Int Immunopharmacol 19: 365-372, 2014

21. Ryan CJ, Aslam M and Courtney JM: Transference of hepatic coma to normal rats from galactosamine treated donors by reverse plasma exchange. Biomater Artif Cells Artif Organs 18 477-482, 1990

22. Pushpavalli G, Kalaiarasi V, Veeramani C and Pugalendi KV: Effect of chrysin on hepatoprotective and antioxidant status in D-galactosamine-induced hepatitis in rats. Eur J Pharmacol 631: 36-41, 2010.

23. Poojari R, Gupta S, Maru G, Khade B and Bhagwat S: Chemopreventive and hepatoprotective effects of embelin on $\mathrm{N}$-nitrosodiethylamine and carbon tetrachloride induced preneoplasia and toxicity in rat liver. Asian Pac J Cancer Prev 11: 1015-1020, 2010.

24. Gavric A, Ribnikar M, Šmid L, Luzar B and Stabuc B: Fat burner induced acute liver injury: Case series of four patients. Nutrition 47: 110-114, 2018

25. Chen B, Jiang L, Hao K, Wang L, Wang Y, Xie Y, Shen J, Zhu M, Tong X, Li K and Wang Z: Protection of plasma transfusion against lipopolysaccharide/d-galactosamine-induced fulminant hepatic failure through inhibiting apoptosis of hepatic cells in mice. J Zhejiang Univ Sci B 19: 436-444, 2018.

26. Giorgi C, Bonora M, Sorrentino G, Missiroli S, Poletti F, Suski J, Galindo Ramirez F, Rizzuto R, Di Virgilio F, Zito E, et al: p53 at the endoplasmic reticulum regulates apoptosis in a $\mathrm{Ca}^{2+}$-dependent manner. Proc Natl Acad Sci USA 112: 1779-1784, 2015.
27. Leifeld L, Nattermann J, Fielenbach M, Schmitz V, Sauerbruch T and Spengler U: Intrahepatic activation of caspases in human fulminant hepatic failure. Liver Int 26: 872-879, 2006.

28. Budihardjo I, Oliver H,Lutter M, Luo X and Wang X: Biochemical pathways of caspase activation during apoptosis. Annu Rev Cell Dev Biol 15: 269-290, 1999.

29. Nakagawa T, Zhu H, Morishima N, Li E, Xu J, Yankner BA and Yuan J: Caspase-12 mediates endoplasmic-reticulum-specific apoptosis and cytotoxicity by amyloid-beta. Nature 403: 98-103, 2000.

30. Heather H, Kenneth H, Samik G and Tung KC: Construction and analysis of a modular model of caspase activation in apoptosis. Theor Biol Med Model 5: 26, 2008.

31. Grosse L, Wurm CA, Brüser C, Neumann D, Jans DC and Jakobs S: Bax assembles into large ring-like structures remodeling the mitochondrial outer membrane in apoptosis. EMBO J 35: 402-413, 2016.

32. Tan CT, Zhou QL, Su YC, Fu NY, Chang HC, Tao RN, Sukumaran SK, Baksh S, Tan YJ, Sabapathy K, et al: MOAP-1 mediates Fas-induced apoptosis in liver by facilitating tBid recruitment to mitochondria. Cell Rep 16: 174-185, 2016.

33. Lv H, Qi Z, Wang S, Feng H, Deng X and Ci X: Asiatic acid exhibits anti-inflammatory and antioxidant activities against lipopolysaccharide and d-galactosamine-induced fulminant hepatic failure. Front Immunol 8: 785, 2017.

34. Korhonen R, Lahti A, Kankaanranta $\mathrm{H}$ and Moilanen E: Nitric oxide production and signaling in inflammation. Curr Drug Targets Inflamm Allergy 4: 471-479, 2005.

35. Rockey DC and Chung JJ: Reduced nitric oxide production by endothelial cells in cirrhotic rat liver: Endothelial dysfunction in portal hypertension. Gastroenterology 114: 344-351, 1998.

36. Shah V, Toruner M, Haddad F, Cadelina G, Papapetropoulos A, Choo K, Sessa WC and Groszmann RJ: Impaired endothelial nitric oxide synthase activity associated with enhanced caveolin binding in experimental cirrhosis in the rat. Gastroenterology 117: 1222-1228, 1999.

37. Petermann H, Vogl S, Schulze E and Dargel R: Chronic liver injury alters basal and stimulated nitric oxide production and $3 \mathrm{H}$-thymidine incorporation in cultured sinusoidal endothelial cells from rats. J Hepatol 31: 284-292, 1999.

38. Sass G, Koerber K, Bang R, Guehring H and Tiegs G: Inducible nitric oxide synthase is critical for immune-mediated liver injury in mice. J Clin Invest 107: 439-447, 2001.

39. Huang CC, Lin KJ, Cheng YW, Hsu CA, Yang SS and Shyur LF: Hepatoprotective effect and mechanistic insights of deoxyelephantopin, a phyto-sesquiterpene lactone, against fulminant hepatitis. J Nutr Biochem 3: 516-530, 2013.

40. Kim YI, Park SW, Yoon YK, Lee KW, Lee JH, Woo HJ and Kim Y: Orostachys japonicus inhibits the expression of MMP-2 and MMP-9 mRNA and modulates the expression of iNOS and COX-2 genes in human PMA-differentiated THP-1 cells via inhibition of NF- $\kappa \mathrm{B}$ and MAPK activation. Mol Med Rep 12: 657-662, 2015.

41. Gao K, Liu F, Guo H, Li J, Zhang Y and Mo Z: miR-224 suppresses HBV replication posttranscriptionally through inhibiting SIRT1-mediated autophagy. Int J Clin Exp Pathol 11: 189-198, 2018.

42. Reddy PV, Murthy ChR and Reddanna P: Fulminant hepatic failure induced oxidative stress in nonsynaptic mitochondria of cerebral cortex in rats. Neurosci Lett 368: 15-20, 2004.

43. Wang Y, Wu Y, Wang B, Xu H, Mei X, Xu X, Zhang X, Ni J and Li W: Bacillus amyloliquefaciens SC06 protects mice against high-fat diet-induced obesity and liver injury via regulating host metabolism and gut microbiota. Front Microbiol 10: 1161, 2019.

This work is licensed under a Creative Commons Attribution-NonCommercial-NoDerivatives 4.0 International (CC BY-NC-ND 4.0) License. 